\title{
A novel role for small molecule glycomimetics in the protection against lipid- induced endothelial dysfunction: involvement of Akt/eNOS and Nrf2/ARE signaling
}

\author{
Ayman M. Mahmoud ${ }^{1,3}$, Fiona L. Wilkinson ${ }^{1}$, Alan M. Jones ${ }^{2}$, James A. Wilkinson ${ }^{4}$, Miguel \\ Romero $^{5}$, Juan Duarte ${ }^{5}$, M. Yvonne Alexander ${ }^{1}$
}

${ }^{1}$ Cardiovascular Research Group, Healthcare Science Research Centre \& Manchester Academic Health Science Centre, UK, ${ }^{2}$ Division of Chemistry and Environmental Science, Faculty of Science and Engineering, Manchester Metropolitan University, ${ }^{3}$ Physiology Division, Department of Zoology, Faculty of Science, Beni-Suef University, Egypt; ${ }^{4}$ Centre for Molecular Drug Design, University of Salford, UK; ${ }^{5}$ Department of Pharmacology, School of Pharmacy, University of Granada, \& Instituto de Investigación Biosanitaria de Granada (Ibs.GRANADA), Spain.

Short title: Mahmoud; Glycomimetics protect endothelial cells.

\section{Corresponding Author:}

Professor M Yvonne Alexander

Healthcare Science Research Centre,

Manchester Metropolitan University,

John Dalton Building,

Chester Street,

Manchester, M15GD. UK.

y.alexander@mmu.ac.uk

+441612475428 


\section{ABSTRACT}

Background: Glycomimetics are a diverse array of saccharide-inspired compounds, designed to mimic the bioactive functions of glycosaminoglycans. Therefore, glycomimetics represent a unique source of novel therapies to target aberrant signaling and protein interactions in a wide range of diseases. We investigated the protective effects of four newly synthesized small molecule glycomimetics against lipid-induced endothelial dysfunction, with an emphasis on nitric oxide (NO) and oxidative stress. Methods: Four aromatic sugar mimetics were synthesized by the stepwise transformation of 2,5-dihydroxybenzoic acid to derivatives (C1-C4) incorporating sulfate groups to mimic the structure of heparan sulfate. Results: Glycomimetic-treated human umbilical vein endothelial cells (HUVECs) were exposed to palmitic acid to model lipid-induced oxidative stress. Palmitate-induced impairment of NO production was restored by the glycomimetics, through activation of Akt/eNOS signaling. Furthermore, C1-C4 significantly inhibited palmitate-induced reactive oxygen species (ROS) production, lipid peroxidation, and activity and expression of NADPH oxidase. These effects were attributed to activation of the Nrf2/ARE pathway and downstream activation of cellular antioxidant and cytoprotective proteins. In ex vivo vascular reactivity studies, the glycomimetics (C1-C4) also demonstrated a significant improvement in endotheliumdependent relaxation and decreased ROS production and NADPH oxidase activity in isolated mouse thoracic aortic rings exposed to palmitate. Conclusions: The small molecule glycomimetics, $\mathrm{C} 1-\mathrm{C} 4$, protect against lipid-induced endothelial dysfunction through up-regulation of Akt/eNOS and Nrf2/ARE signaling pathways. Thus, carbohydrate-derived therapeutics are a new class of glycomimetic drugs targeting endothelial dysfunction, regarded as the first line of defense against vascular complications in cardiovascular disease.

\section{Key words:}

Small molecule drug discovery, glycomimetics, heparan sulfate, endothelial dysfunction, oxidative stress. 


\section{INTRODUCTION}

The vascular endothelium plays a pivotal role in regulating vascular tone, controlling tissue blood flow, vessel integrity, permeability, inflammatory responses, vascular remodeling and angiogenesis [1, 2]. Pathological conditions can be detrimental to these processes and initiate endothelial dysfunction resulting in the development of vascular complications $[3,4]$, characterized by the reduced bioavailability of nitric oxide (NO), which is critical in maintaining the physiological function of vasculature [5]. In addition, an imbalance between anti-thrombotic and pro-thrombotic factors predisposes the endothelium to an atherogenic milieu, contributing to endothelial dysfunction [6]. Thus, endothelial dysfunction has emerged as a critical early target for preventing atherosclerosis and cardiovascular disease [7].

Endothelial dysfunction is linked to the pathogenesis of common diseases associated with increased risk of CVD, including type II diabetes mellitus, obesity and metabolic syndrome [8]. It has been reported that chronic elevations in plasma free fatty acids (FFAs) contribute to the development of endothelial damage, diminished endothelium-dependent vasodilation, and hypertension $[9,10]$ by triggering overproduction of reactive oxygen species (ROS), leading to uncoupling of eNOS [11]. Palmitic acid, the most prominent FFA in the bloodstream, stimulates the production of ROS, at least in part, through activating nicotinamide adenine dinucleotide phosphate (NADPH) oxidases and down-regulation of eNOS and associated protective pathways, including nuclear factor-erythroid 2-related factor 2 (Nrf2) $[12,13]$. Decreasing lipotoxicity and its associated oxidative stress, as well as promoting up-regulation of anti-oxidant enzymes and cytoprotective proteins, may be key components to prevent and treat cardiovascular diseases.

The glycosaminoglycan, (GAG) heparan sulfate (HS), is highly sulfated and key to a variety of physiological processes, including wound healing, angiogenesis, inflammation and development, [14] often acting as a co-receptor for signaling pathways. The multiple biological functions of HS are attributed to their variable sulfation patterning, which in turn, allows for diverse but specific ionic interactions between the negatively charged sulfates and the carboxylate groups of a variety of proteins, including proteases, 
growth factors and cytokines [14-16]. HS oligosaccharides can mimic HS functions in biological systems; however, their use has been hindered by the complexity of their synthesis [17]. Thus, small molecule mimics; glycomimetics, can offer a potential solution to this obstacle [18].

To the best of our knowledge, there are no published reports regarding the possible protective effects of small molecule HS mimetics against lipid-induced endothelial dysfunction. Here, we demonstrate the evaluation of a class of small molecule glycomimetics, establishing for the first time their protective effects against palmitate-induced endothelial dysfunction, using in vitro and ex vivo models. This study provides an understanding of the mechanisms underlying the vascular protective effects of glycomimetics, in terms of oxidative stress and endothelial function and their potential as a new class of therapeutic drugs to target endothelial dysfunction.

\section{MATERIALS AND METHODS}

\section{Synthesis of small molecule glycomimetics}

Glycomimetics were synthesized by the stepwise transformation of 2,5-dihydroxybenzoic acid to a range of 2,5-substituted benzoic acid derivatives incorporating the key sulfate groups to mimic the interactions of HS (Supplementary Fig. I). Namely, commercially available 2,5-dihydroxybenzoic acid was converted to the methyl ester using Fischer esterification conditions [19]. In turn, the methyl 2,5-dihydroxybenzoate can be mono or di-allylated to afford methyl 5-(allyloxy)-2-hydroxybenzoate or methyl 2,5bis(allyloxy)benzoate, respectively [20]. Methyl 5-(allyloxy)-2-hydroxybenzoate can be further alkylated with 4-bromobutyl acetate to afford methyl 2-(4-acetoxybutoxy)-5-(allyloxy)benzoate. Either as the methyl ester or carboxylic acid the above olefin-containing intermediates were treated with either AD-mix- $\alpha$ or $\beta$ under Sharpless asymmetric dihydroxylation [21] conditions to afford the chiral di or tetra-hydroxylated compounds. Finally, treatment with sulfur trioxide-trimethylamine complex gave the desired HS-mimics (C1-C4). The chemical methods are described in detail in the Supplementary material online. In total, 4 
examples of glycomimetics (C1-C4) have been prepared, the structures of which are shown in Figure 1. The molecular weights of C1-C4 range from 554-636 Da, total polar surface area (tPSA) are all $\geq 235 \mathrm{~A}^{2}$, calculated $\log \mathrm{P}($ octanol/water solubility $) \leq-0.13$ and hydrogen bond acceptors $\geq 16$, however the number of hydrogen bond donating groups are allowable at $\leq 5$, therefore, these compounds fall outside the Lipinski's rule of 5 (Ro5) [22], for orally bioavailable drug molecules (Supplementary Table I). This is due to the necessary high level of sulfation to mimic HS, but act as a useful starting point for the discovery of repurposed glycomimetics.

\section{Human umbilical vein endothelial cell (HUVEC) culture}

HUVECs (Caltag, Medsystems, Buckingham, UK) from passage 2-7, were cultured in media (M199; Lonza, Belgium) containing 20\% fetal bovine serum (FBS), penicillin/streptomycin ( $2 \mathrm{mmol} / \mathrm{L})$, glutamine ( $2 \mathrm{mmol} / \mathrm{L})$, HEPES $(10 \mathrm{mmol} / \mathrm{L})$, endothelial cell growth supplement $(30 \mu \mathrm{g} / \mathrm{mL})$ and heparin $(100 \mu \mathrm{g} / \mathrm{mL})$ under $5 \% \mathrm{CO}_{2}$ at $37^{\circ} \mathrm{C}$.

\section{Cell viability}

HUVECs were seeded in $96-$ well microtiter plate and incubated at $37^{\circ} \mathrm{C}$ for 24 hours. The medium was removed, replaced by fresh medium and then exposed to serial dilutions of C1-C4 (1, 10, 100 and 500 $\mu \mathrm{mol} / \mathrm{L})$ for 24 hours. After incubation, $20 \mu \mathrm{L}$ of $5 \mathrm{mg} / \mathrm{mL}$ MTT (3-(4,5-dimethylthiazol-2-yl)-2,5diphenyltetrazolium bromide) in phosphate buffered saline (PBS) was added to the cells and further incubated at $37^{\circ} \mathrm{C}$ for $15 \mathrm{~min}$. After washing, $100 \mu \mathrm{L}$ of DMSO were added to each well and absorbance was measured at $570 \mathrm{~nm}$ on a microplate reader (BioTek) with subtraction of the blank value at $630 \mathrm{~nm}$, and compared with control untreated cells.

\section{Preparation of palmitate-albumin complexes and cell treatments}

To generate the in vitro model of disease, sodium palmitate was conjugated to bovine serum albumin (BSA) as outlined by Chavez and Summers [23], with some modifications. Sodium palmitate was 
dissolved in ethanol at $60^{\circ} \mathrm{C}$ and diluted 1:100 in M199 media supplemented with $2 \%$ fatty acid-free bovine serum albumin (BSA) and incubated for 1 hour at $37^{\circ} \mathrm{C}$. HUVECS were treated with $1 \mu \mathrm{mol} / \mathrm{L}$ of the synthesized glycomimetics (C1-C4) in the presence of palmitate $(100 \mu \mathrm{mol} / \mathrm{L})$ for 3 hours or pre-treated with glycomimetics for 12 hours followed by 3 hours palmitate $(100 \mu \mathrm{mol} / \mathrm{L})$. In some experiments, HUVECs were incubated with 1,10 or $100 \mu \mathrm{mol} / \mathrm{L}$ of the test compounds (C1-C4). Control cells were incubated in serum free M199 containing 2\% (wt/vol) fatty acid-free BSA.

\section{NO release assay}

NO release was determined using, diaminofluorescein-2 (DAF-2) [24]. Briefly, cells were washed with PBS and incubated with L-arginine $(100 \mu \mathrm{mol} / \mathrm{L} / \mathrm{PBS})$ for $5 \mathrm{~min}$ at $37^{\circ} \mathrm{C}$ before incubating with DAF-2 $(0.1 \mu \mathrm{mol} / \mathrm{L})$ for $2 \mathrm{~min}$, followed by the calcium ionophore calimycin $(\mathrm{A} 23187,1 \mu \mathrm{mol} / \mathrm{L})$ for 30 minutes. L-NAME $(100 \mu \mathrm{mol} / \mathrm{L})$, a nitric oxide inhibitor (NOS), was also added $20 \mathrm{~min}$ before the addition of Larginine in some experiments to demonstrate the involvement of NOS. DAF-2 fluorescence (arbitrary units, AU) was quantified using a microplate reader (BioTek) with excitation and emission wavelengths of 485 and 540, respectively. The autofluorescence was subtracted from each value.

\section{Quantification of ROS production}

Cells were incubated with $10 \mu \mathrm{mol} / \mathrm{L}$ of the fluorescent probe 2'-7'-dichlorodihydrofluorescein diacetate $\left(\mathrm{CM}-\mathrm{H}_{2} \mathrm{DCFDA}\right.$, Sigma) for $30 \mathrm{~min}$ at $37^{\circ} \mathrm{C}$ and the level of fluorescence was determined (excitation 490 $\mathrm{nm}$; emission $540 \mathrm{~nm}$ ) using a microplate reader (BioTek).

\section{NADPH oxidase activity}

The level of NADPH-enhanced superoxide anions $\left(\mathrm{O}_{2}{ }^{--}\right)$in HUVEC homogenates or intact mouse aortic rings was determined by lucigenin-enhanced chemiluminescence, as demonstrated previously [25]. Following the various treatment conditions, cells or aortic rings were homogenized in a buffer $(20 \mathrm{mmol} / \mathrm{L}$ $\mathrm{KH}_{2} \mathrm{PO}_{4}$ and $1 \mathrm{mmol} / \mathrm{L}$ EDTA supplemented with aprotinin, leuprotinin, pepstatin and PMSF) and the total 
protein quantified using the Bicinchoninic acid (BCA) assay (Pierce Biotechnology). To $50 \mu \mathrm{g}$ of total protein, NADPH $(100 \mu \mathrm{mol} / \mathrm{L})$ was added in a total volume of $500 \mu \mathrm{l}$, with lucigenin $(5 \mu \mathrm{mol} / \mathrm{L})$ injected automatically. Activity was determined by measuring luminescence over $200 \mathrm{~s}$ in a scintillation counter (Lumat LB 9507, Berthold, Germany). Basal values of NADPH oxidase activity were subtracted from the experimental values and expressed as RLU/min/ $/ \mu$ g protein for cells or RLU/min/mg tissue for aortic rings.

\section{Lipid peroxidation assay}

The OxiSelect TBARS assay kit (Cell Biolabs, San Diego, USA) was used to determine the level of lipid peroxidation by measuring malondialdehyde (MDA). After treatment, HUVECs were washed with PBS and homogenized in RIPA buffer. SDS lysis solution was added to the samples and MDA standards followed by incubation with thiobarbituric acid for 45 minutes at $95^{\circ} \mathrm{C}$. Samples were cooled to room temperature and centrifuged at $1000 \mathrm{~g}$ for $15 \mathrm{~min}$. The supernatant was removed and the absorbance was measured at $532 \mathrm{~nm}$ using a microplate reader (BioTek). Lipid peroxidation was expressed as nmol $\mathrm{MDA} / \mathrm{mg}$ protein.

\section{Superoxide dismutase (SOD) and catalase (CAT) activity assays}

The activity of the enzymes, SOD and CAT, was determined in cell homogenates using assay kits (Cayman; Cat No 706002 and 707002 respectively), according to the manufacturer's instructions. A unit of SOD was defined as the amount of enzyme required to demonstrate $50 \%$ dismutation of superoxide radicals. Similarly, a unit of CAT was defined as the amount required to the form $1.0 \mathrm{nmol}$ of formaldehyde per min at $25^{\circ} \mathrm{C}$.

\section{Quantitative reverse transcriptase-polymerase chain reaction (RT-PCR) analysis}

Trizol (Invitrogen) was used to isolate total RNA from HUVECs and was quantified at $260 \mathrm{~nm}$ using a Nanodrop. RNA samples with A260/A280 ratios < 1.7 were discarded. cDNAs were synthesized from total 
RNA (2 $\mu \mathrm{g})$, using an oligo deoxythymidine primer and SuperScript II reverse transcriptase (Sigma) and were amplified using the SYBR Green master mix (Bioline, UK) in a total volume of $20 \mu 1$ (Surecycler 8800 thermocycler; Agilent Technologies) with the primer sets outlined in Supplementary Table II. Realtime PCR was performed using the following conditions; $10 \mathrm{~min}$ at $95^{\circ} \mathrm{C}$, followed by 40 cycles of $30 \mathrm{sec}$ at $95^{\circ} \mathrm{C}, 60 \mathrm{sec}$ at annealing temperature of respective primer set, and extension for $30 \mathrm{sec}$ at $72^{\circ} \mathrm{C}$. PCR products were assessed for quality using standard melting curve analysis and an agarose gel electrophoresis. The cycle threshold (CT) values were analyzed using the $2^{-\Delta \Delta \mathrm{Ct}}$ method and data normalized to GAPDH.

\section{Western blot analysis}

HUVECs were lysed in RIPA buffer, containing proteinase inhibitors and total protein quantified using BCA protein assay kit (Pierce Biotechnology). Proteins (30 $\mu \mathrm{g} / \mathrm{well})$ were denatured and separated by sodium dodecyl sulphate (SDS)-polyacrylamide gel electrophoresis and transferred to polyvinylidene difluoride (PVDF) membranes. Blots were blocked for 1 hour in 5\% non-fat milk/Tris-Buffered Saline Tween-20 (TBS-T), probed with primary rabbit anti-phospho-protein kinase B (Akt), rabbit anti-Akt (Santa Cruz Biotechnology), rabbit polyclonal anti- phospho-eNOS (Cell Signaling), rabbit anti-eNOS, rabbit antiHO-1 (Stressgen), rabbit anti-Nrf2, rabbit anti-NQO-1 (Santa Cruz Biotechnology) or mouse anti- $\beta$-actin (Sigma), overnight at $4^{\circ} \mathrm{C}$ followed by washing and incubation with the corresponding horse radish peroxidase-labelled secondary antibody for 1 hour at room temperature. After washing with TBS-T, membranes were incubated with ECL reagent and visualized (Amersham Pharmacia Biotech). Densitometry analysis using ImageJ (version 1.32j, NIH, http://rsb.info.nih/ij/) was used to quantify protein signal.

\section{Ex vivo vascular reactivity}


Thoracic aortas from the experimental male BALB/c mice (Janvier, St Berthevin Cedex, France) were harvested, cleared of periadventitial tissue and cut transversely into 2.0-mm rings. All the procedures conform to the Guide for the Care and Use of Laboratory Animals (National Institutes of Health (NIH) publication no. 85-23, revised 2011) and approved by the Institutional Committee for the ethical care of animals (University of Granada, Spain). Mouse thoracic aortic (MTA) rings were maintained in Dulbecco's modified Eagle's medium (DMEM) supplemented with 10\% FBS, and antibiotics (100 U/mL penicillin, and $100 \mu \mathrm{g} / \mathrm{mL}$ streptomycin). MTA rings were then incubated in serum-free DMEM containing $2 \%$ fatty acid-free BSA (control) or palmitate-conjugated BSA $(100 \mu \mathrm{mol} / \mathrm{L})$ in the presence of glycomimetics $(1$ or $10 \mu \mathrm{mol} / \mathrm{L}$ ) for a 24 hour duration. Care was taken with the vascular rings to avoid damage of the endothelial layer and transferred to a chamber filled with fresh Kreb's solution and mounted in a myograph (model 610M, Danish Myo Technology, Aarhus, Denmark) for isometric tension measurement, as described previously [26]. Cumulative dose-response relaxation curves to acetylcholine (ACh; $1 \mathrm{nmol} / \mathrm{L}-10$ $\mu \mathrm{mol} / \mathrm{L})$ were performed in intact rings pre-contracted by U46619 $(10 \mathrm{nmol} / \mathrm{L})$ in control or L-NAME $(100$ $\mu \mathrm{mol} / \mathrm{L})$-treated aortic rings. The mouse aorta were incubated with mitochondrial antioxidant mitoQ $(0.1$ $\mu \mathrm{mol} / \mathrm{L})$ or the NADPH oxidase inhibitor apocynin $(10 \mu \mathrm{mol} / \mathrm{L})$ for $60 \mathrm{~min}$ before the addition of U46619 to establish the source of ROS following palmitate treatment and relaxant responses to ACh were subsequently measured and expressed as a percentage of pre-contraction.

\section{In situ detection of vascular ROS content}

MTA rings were incubated in serum-free DMEM containing 2\% fatty acid-free BSA (control) or palmitate conjugated BSA $(100 \mu \mathrm{mol} / \mathrm{L})$ in the presence of glycomimetics $(1$ or $10 \mu \mathrm{mol} / \mathrm{L})$ for 24 hours. Unfixed MTA rings were cryopreserved in $0.1 \mathrm{~mol} / \mathrm{L}$ PBS plus $30 \%$ sucrose for 1 hour, included in OCT compound medium (Tissue-Tek; Sakura Finetechnical), and kept frozen $\left(-80^{\circ} \mathrm{C}\right)$. By using a cryostat (Microm InternationalModelHM500 OM), $10 \mu \mathrm{m}$ cross sections were obtained and incubated for 30 min in Hepesbuffered solution containing $10 \mu \mathrm{mol} / \mathrm{L}$ dihydroethidium (DHE), which incalates with DNA in the presence of ROS and is detected by fluorescence. Cells were counterstained with the nuclear stain DAPI at 0.3 
$\mu \mathrm{mol} / \mathrm{L}$. In the following 24 hours, sections were examined using a fluorescence microscope (Leica DMIRB,). Sections were photographed and fluorescence was quantified using ImageJ. All parameters (pinhole, contrast, gain and offset) were held constant for all sections from the same experiment. ROS production was estimated from the ratio of ethidium/DAPI fluorescence [27].

\section{Statistical analysis}

Data were analysed using GraphPad Prism 5 (GraphPad Software, San Diego, USA) and comparisons were made using one-way analysis of variance (ANOVA) test and Tukey's post-hoc analysis. Two-way ANOVA tested for group interactions and results were expressed as mean \pm standard error of the mean and $\mathrm{P}=\leq 0.05$ was considered significant.

\section{RESULTS}

\section{Glycomimetics do not affect cell viability}

We tested the effects of glycomimetics C1-C4 (Fig.1) on HUVEC viability using concentrations ranging from 1 to $500 \mu \mathrm{mol} / \mathrm{L}$. After incubation for 24 hours, no detrimental effects on HUVEC viability was detected with any glycomimetic compound at any concentration (Supplementary Fig. II).

\section{Glycomimetics rescue palmitate-induced impairment of NO production}

First, to study the concentration-dependent effect of the synthesized glycomimetics on NO production, HUVECs were incubated with 1, 10 or $100 \mu \mathrm{mol} / \mathrm{L}$ of the test compounds (C1-C4) for 12 hours, followed by 3 hours palmitate $(100 \mu \mathrm{mol} / \mathrm{L})$. C1 significantly increased A23187-stimulated NO production from HUVECs at the $1 \mu \mathrm{mol} / \mathrm{L}(\mathrm{P}<0.05), 10 \mu \mathrm{mol} / \mathrm{L}(\mathrm{P}<0.001)$ and $100 \mu \mathrm{mol} / \mathrm{L}(\mathrm{P}<0.01)$ concentrations (Supplementary Fig. IIIA). Pre-treatment with different doses of $\mathbf{C 2}$ significantly $(\mathrm{P}<0.001)$ protected HUVECS against palmitate-induced reduction in NO (Supplementary Fig. IIIB). The lower dose (1 $\mu \mathrm{mol} / \mathrm{L})$ of $\mathbf{C 3}$ produced a significant $(\mathrm{P}<0.05)$ increase in A23187-stimulated NO production from HUVECs and 
the higher doses $(10$ and $100 \mu \mathrm{mol} / \mathrm{L})$ produced a more potent effect $(\mathrm{P}<0.001$, Supplementary Fig. IIIC). Similar to C1, C4 significantly increased NO production from HUVECs at the $1 \mu \mathrm{mol} / \mathrm{L}(\mathrm{P}<0.05), 10$ $\mu \mathrm{mol} / \mathrm{L}(\mathrm{P}<0.001)$ and $100 \mu \mathrm{mol} / \mathrm{L}(\mathrm{P}<0.01)$ concentrations (Supplementary Fig. IIID). In HUVECs treated with 3 different doses of $\mathbf{C 1}, \mathbf{C 2}, \mathbf{C} 3$ or $\mathbf{C 4}$, the eNOS inhibitor L-NAME significantly $(\mathrm{P}<0.001)$ abolished A23187-stimulated NO production from HUVECS when compared with the corresponding glycomimetic

dose. However, in light of the consistent response with all four compounds, we selected to use the low dose of $1 \mu \mathrm{mol} / \mathrm{L}$ for all future glycomimetic treatments.

Next, to determine the restorative effect of the glycomimetics on palmitate-induced reduction in NO production, HUVECs were incubated either with $1 \mu \mathrm{mol} / \mathrm{L}$ of the synthesized glycomimetics (C1-C4) in the presence of palmitate $(100 \mu \mathrm{mol} / \mathrm{L})$ for 3 hours, or pre-treated with glycomimetics for 12 hours followed by 3 hours palmitate $(100 \mu \mathrm{mol} / \mathrm{L})$ respectively. HUVECs incubated with palmitate, exhibited a significant $(\mathrm{P}<0.001)$ decrease in A23187-stimulated NO production. In HUVECs pre-treated with palmitate, followed by incubation with $\mathbf{C 1}, \mathbf{C 2}, \mathbf{C} 3$ or $\mathbf{C 4}$ for 3 hours, the dramatic decrease in NO production was significantly $(\mathrm{P}<0.001)$ attenuated compared to palmitate controls (Fig. 2A). Pre-incubation with $1 \mu \mathrm{mol} / \mathrm{L}$ C1-C4, significantly protected HUVECs against the significant decline in A23187-stimulated NO production in the presence of palmitate (Fig. 2B).

\section{Glycomimetics modulate mRNA and phosphorylation levels of eNOS and Akt under palmitate-} induced oxidative stress

To determine whether the enhanced NO release observed after treatment with glycomimetics under palmitate-induced stress conditions was due to activation of the Akt/eNOS pathway, both the level of mRNA abundance and protein phosphorylation of Akt and eNOS were investigated. HUVECs were preincubated with $1 \mu \mathrm{mol} / \mathrm{L}$ of the glycomimetics (C1-C4), followed by palmitate for 3 hours. Palmitate treatment alone produced a marked decline in both Akt mRNA $(\mathrm{P}<0.01$, Fig. 3A) and protein phosphorylation (pAkt; $\mathrm{P}<0.05$, Fig. 3B). Pretreatment of HUVECs with $\mathbf{C 1}, \mathbf{C 2}, \mathbf{C 3}$ or $\mathbf{C 4}$ significantly 
$(\mathrm{P}<0.05)$ increased Akt mRNA abundance, and Akt protein phosphorylation, with pAkt restored to untreated control levels. A more marked effect in terms of Akt phosphorylation $(\mathrm{P}<0.01)$ was observed with C4.

Similarly, palmitate significantly reduced eNOS mRNA abundance $(\mathrm{P}<0.01$, Fig. $3 \mathrm{C})$ and protein phosphorylation (peNOS; $<<0.05$, Fig. 3D) in HUVECs treated for 3 hours. eNOS mRNA abundance exhibited a marked increase in HUVECS pretreated with $\mathbf{C 1}(\mathrm{P}<0.05), \mathbf{C 2}(\mathrm{P}<0.01), \mathbf{C} 3(\mathrm{P}<0.05)$ or $\mathbf{C 4}$ $(\mathrm{P}<0.05)$. There was a significant increase in eNOS protein phosphorylation in HUVECs pretreated with $\mathbf{C 1}(\mathrm{P}<0.05), \mathbf{C 2}(\mathrm{P}<0.05), \mathbf{C} 3(\mathrm{P}<0.05)$ or $\mathbf{C 4}(\mathrm{P}<0.001)$ followed by palmitate treatment, compared to palmitate-treated controls, with the greatest effect observed with C4. Again, peNOS was restored to untreated control levels when pre-treated with all four glycomimetics.

\section{Glycomimetics attenuate palmitate-induced ROS production and oxidative stress}

Next, we investigated the effect of the glycomimetics on palmitate-induced oxidative stress by assessing ROS production, lipid peroxidation and activity of the antioxidant enzymes, SOD and CAT. HUVECs treated with palmitate for 3 hours exhibited a significant $(\mathrm{P}<0.001)$ increase in ROS production as expected. This ROS induction was significantly reduced $(\mathrm{P}<0.001)$ after co-treatment with $1 \mu \mathrm{mol} / \mathrm{L}$ of $\mathbf{C 1}, \mathbf{C 2}, \mathbf{C 3}$ or $\mathbf{C 4}$ to untreated control levels (Fig. 4A). The same protective effect was apparent when pre-treated with C1-C4 for 12 hours $(\mathrm{P}<0.001$; Fig. 4B. The concentration-dependent effect of the glycomimetic compounds C1-C4 on palmitate-induced ROS production was also studied in the pre-treatment model. All compounds at the different doses $(1,10$ or $100 \mu \mathrm{mol} / \mathrm{L})$ significantly abolished the palmitate-induced ROS production to the same extent and to untreated control levels (Supplementary Fig. IV).

Supporting the ROS data, lipid peroxidation (assayed as MDA) showed a significant $(\mathrm{P}<0.001)$ increase in HUVECs treated with palmitate for 3 hours which was significantly reduced $(\mathrm{P}<0.05)$ after pretreatment with $1 \mu \mathrm{mol} / \mathrm{L}$ C1-C4, (Fig. 4C). 
The activity of the anti-oxidant enzyme SOD was, as expected significantly $(\mathrm{P}<0.01)$ decreased in HUVECs treated with palmitate for 3 hours, an effect which was prevented when pre-treated with glycomimetic $\mathbf{C 1}$ $(\mathrm{P}<0.05), \mathbf{C 2}(\mathrm{P}<0.001), \mathbf{C 3}(\mathrm{P}<0.001)$ or $\mathbf{C 4}(\mathrm{P}<0.01$; Fig. 4D). CAT activity exhibited a similar pattern where it was significantly $(\mathrm{P}<0.001)$ lower in palmitate treated cells and restored to untreated control levels in HUVECs pretreated with $\mathbf{C 1}(\mathrm{P}<0.01), \mathbf{C 2}(\mathrm{P}<0.01), \mathbf{C 3}(\mathrm{P}<0.001)$ or $\mathbf{C 4}(\mathrm{P}<0.05)$ glycomimetics, with C3 showing the most marked effect (Fig. 4E).

\section{Glycomimetics reduce palmitate-stimulated NADPH oxidase activity}

To further validate the protective effect of the glycomimetic compounds C1-C4 against palmitate-induced oxidative stress, NADPH oxidase activity and mRNA expression of its subunits were assayed. Palmitate induced a significant $(\mathrm{P}<0.001)$ increase in NADPH oxidase activity when compared with untreated control HUVECs. Pretreatment with $1 \mu \mathrm{mol} / \mathrm{L}$ C1-C4 significantly $(\mathrm{P}<0.001)$ decreased NADPH oxidase activity in palmitate-treated HUVECs (Fig. 5A). Furthermore, HUVECs treated with palmitate for 3 hours exhibited a significant increase in mRNA abundance of the NADPH oxidase subunits $\mathrm{p} 22^{\text {phox }}(\mathrm{P}<0.001$; Fig. 5B), NOX1 ( $\mathrm{P}<0.001$; Fig. 5C), NOX4 ( $\mathrm{P}<0.01$; Fig. 5D), and $\mathrm{p} 47^{\text {phox }}(\mathrm{P}<0.001$; Fig. 5E). However, in the

presence of all 4 glycomimetics, a significant protective effect was detected with a down-regulation of mRNA abundance of NOX1, NOX4, p4 $7^{\text {phox }}$ and $\mathrm{p} 22^{\text {phox }}$ (Figure 5, $\mathrm{P}<0.05$ ) compared to palmitate alone.

\section{Glycomimetics protect against palmitate-induced oxidative stress via the Nrf2/ARE pathway}

To establish the downstream protective anti-oxidant effects of the glycomimetic compounds C1-C4, against palmitate-induced oxidative stress, mRNA and protein expression of Nrf2, NQO-1 and HO-1 were interrogated using qRT-PCR and western blotting, respectively (Fig. 6).

Under palmitate-induced oxidative stress conditions, a significant reduction in the mRNA abundance and protein levels of Nrf2, NQO-1 and HO-1 was observed, when compared to homeostatic controls (Fig. 6). 
Palmitate-induced HUVECs exhibited a significant up-regulation and restoration of Nrf2, NQO-1 and HO1 mRNA abundance when pretreated with $\mathbf{C 1}(\mathrm{P}<0.001)$, $\mathbf{C} 2(\mathrm{P}<0.01), \mathbf{C} 3(\mathrm{P}<0.01)$ or $\mathbf{C 4}(\mathrm{P}<0.001)$ (Fig. 6). In support of the mRNA data, a correlation was found with, Nrf2, NQO-1 and HO-1 protein expression where we demonstrate a significant increase in HUVECs pretreated with $\mathbf{C 1}(\mathrm{P}<0.05), \mathbf{C 2}(\mathrm{P}<0.01), \mathbf{C 3}$ $(\mathrm{P}<0.001)$ or $\mathbf{C 4}(\mathrm{P}<0.05)$, with $\mathbf{C} 2$ and $\mathbf{C} 3$ exhibiting the most potent effects (Fig. 6).

\section{Glycomimetics restore endothelium-dependent vasodilatation ex vivo after palmitate treatment}

In order to establish whether these effects could be validated in a more physiological model, an ex vivo contractility study was conducted to evaluate the effect of C1-C4 treatment on the endothelium-dependent vasodilatation (Fig. 7). Mouse aortic rings were incubated with $100 \mu \mathrm{mol} / \mathrm{L}$ palmitate and 1 or $10 \mu \mathrm{mol} / \mathrm{L}$ of C1-C4 for 24 hours and concentration-relaxation response curves to ACh were performed in intact rings pre-contracted by the prostaglandin analog, U46619.

Aortas treated with palmitate showed a significant $(\mathrm{P}<0.001)$ decrease in endothelium-dependent vasodilator responses to $\mathrm{ACh}$, compared with the control aortas. $\mathbf{C 1}$ and $\mathbf{C 2}$, at both doses (1 and 10 $\mu \mathrm{mol} / \mathrm{L})$, significantly $(\mathrm{P}<0.001)$ improved the palmitate-reduced endothelium-dependent vasodilatation (Fig. 7A and B, respectively). Both doses of $\mathbf{C 3}$ produced a significant $(\mathrm{P}<0.01)$ increase in endotheliumdependent vasodilatation when compared with the palmitate-induced aortas (Fig. 7C). However, the improved endothelium-dependent vasodilatation produced by $\mathbf{C} 3$ treatment was significantly $(\mathrm{P}<0.05)$ lower than the control aortas. The lower concentration of $\mathbf{C 4}(1 \mu \mathrm{mol} / \mathrm{L})$ showed a marked $(\mathrm{P}<0.01)$ alleviation in endothelium-dependent vasodilatation, but was still significantly $(\mathrm{P}<0.01)$ lower than the control aortas (Fig. 7D). The higher dose of $\mathbf{C 4}(10 \mu \mathrm{mol} / \mathrm{L})$ significantly $(\mathrm{P}<0.001)$ improved endotheliumdependent vasodilatation when compared with the palmitate-induced aortas and was similar to untreated controls. 
To validate the endothelial-dependent relaxation responses to ACh, the eNOS inhibitor L-NAME was added, which abolished the relaxant response induced by ACh in all experimental groups as shown in Supplementary Figure V. To examine whether ROS are involved in endothelial dysfunction induced by palmitate in mouse aorta, the endothelium-dependent relaxant response to ACh in the presence of the mitochondrial antioxidant mitoQ (Fig. 7E) or the NADPH oxidase inhibitor apocynin were analyzed (Fig. 7F). Both mitoQ and apocynin significantly improved the impaired aortic relaxation in response to ACh induced by palmitate, suggesting involvement of ROS-induced pathways.

\section{Glycomimetics attenuate palmitate-induced vascular ROS levels by reducing NADPH oxidase activity ex vivo}

To characterize and localize ROS levels within the vascular wall, aortic rings were incubated with 100 $\mu \mathrm{mol} / \mathrm{L}$ palmitate and 1 or $10 \mu \mathrm{mol} / \mathrm{L}$ of $\mathbf{C 1}-\mathbf{C 4}$ for 24 hours and the level of red fluorescence was determined in sections of aorta incubated with DHE (Fig. 8A). Rings treated with palmitate showed significantly $(\mathrm{P}<0.001)$ increased staining in adventitial, medial and endothelial cells compared with the control group. Sections from palmitate-treated rings exhibited a marked decrease in red fluorescence $(\mathrm{P}<0.01)$ when co-incubated with any glycomimetic at either dose, suggesting attenuation of ROS production.

NADPH oxidase activity measured by lucigenin-enhanced chemiluminescence (Fig. 8B) was significantly $(\mathrm{P}<0.001)$ increased by palmitate. Co-incubation of aortic rings with either dose of $\mathbf{C} 1$ or $\mathbf{C} 2$ significantly $(\mathrm{P}<0.001)$ reduced NADPH oxidase activity when compared to palmitate alone. $\mathbf{C 3}$ significantly decreased NADPH oxidase activity at both the $1(\mathrm{P}<0.01)$ and $10 \mu \mathrm{mol} / \mathrm{L}(\mathrm{P}<0.001)$ doses. $\mathbf{C} 4$ exhibited a similar pattern as it significantly $(\mathrm{P}<0.01)$ reduced NADPH oxidase activity at both applied concentrations.

\section{DISCUSSION}


Glycosaminoglycans represent the most abundant class of molecules in nature. They are involved in disease indications; however, they lack the properties necessary for efficacious drugs. Therefore, design and synthesis of small molecule glycomimetics, exhibiting drug-like properties could represent a new class of therapeutic drugs to target molecular mechanisms of disease $[18,28]$. Here, we report the discovery and evaluation of the effects of glycomimetics in a FFA-induced model of endothelial dysfunction. We demonstrate that glycomimetics exert protective effects against palmitate-induced endothelial dysfunction. Our data point to the involvement of Akt/eNOS and Nrf2/ARE signaling pathways in mediating these protective effects on the endothelium.

HUVECs were used in both a pre-treatment and co-incubation regime to reflect a potential preventative mechanism and possible treatment of disease in progress respectively. Palmitate-treated HUVECs showed diminished A23187-stimulated NO production mediated via reduced Akt-dependent phosphorylation of eNOS at Ser1177. This observation confirms previous work demonstrating palmitate induced eNOS uncoupling, decreased eNOS phosphorylation and ultimately NO production $[11,26]$. We show that small molecule glycomimetics markedly prevented the palmitate-induced decline in NO production through upregulation of the mRNA abundance and protein phosphorylation of both Akt and eNOS.

The FFA-induced decline in NO production could be explained by the overproduction of ROS. Palmitic acid stimulates the production of superoxide radicals through activating NADPH oxidases and downregulation of eNOS $[12,13]$. Superoxide reacts with NO, forming the potent and versatile oxidant peroxynitrite $(\mathrm{ONOO} \bullet-)$ which may oxidize the eNOS cofactor tetrahydrobiopterin or destabilize the eNOS dimer, causing uncoupling of eNOS [29]. Uncoupled eNOS produces superoxide rather than NO, thus further augmenting oxidative stress and decreasing NO bioavailability [29]. In addition, ROS activates membrane oxidases leading to increased dimethylarginine, an arginine decoy for the active sites on eNOS and L-arginine transporters [30]. Here, palmitate induced a marked increase in ROS production through activating NADPH oxidase, as previously reported [26, 31, 32]. In addition, we demonstrated increased expression of the NADPH oxidase subunits, NOX1, NOX4, p22phox and p47phox, in palmitate-induced 
HUVECS. The formation of ROS is further aggravated by reduced antioxidant defenses, leading to oxidative damage and endothelial cell dysfunction [33]. The intracellular antioxidant system includes SOD and CAT that metabolize toxic oxidative stress intermediates; SOD catalyzes the conversion of superoxide to $\mathrm{H}_{2} \mathrm{O}_{2}$ and $\mathrm{O}_{2}$ and CAT converts $\mathrm{H}_{2} \mathrm{O}_{2}$ to water [34] and given that we demonstrate a significant restoration of SOD and CAT activity in the presence of $\mathrm{C} 1-\mathrm{C} 4$, we propose that reducing ROS production by these glycomimetic compounds act in the management of FFA-induced endothelial dysfunction and vascular complications.

Small molecule glycomimetics were able to reduce ROS production through decreasing NADPH oxidase activity and down-regulating the expression of its subunits. Moreover, the tested glycomimetics decreased lipid peroxidation, an important intermediary process in oxidative stress, and ameliorated intracellular redox status, as shown by the enhanced activity of SOD and CAT. The potentiation of antioxidant defenses and normalization of ROS would appear to contribute to the restoration of endothelial function.

To understand the molecular basis for the protective effects of the glycomimetics, we hypothesized a specific mechanism that could be associated to their potential, to elicit cell adaptive responses involving the transcription factor nuclear factor-erythroid 2-related factor 2 (Nrf2). Nrf2 is a ubiquitous transcription factor, which plays an important role in cellular resistance to oxidative stress. The Nrf2 protein is sequestered in the cytosol, by the actin-binding protein, Kelch-like ECH-associated protein 1 (Keap1). Under physiological conditions, Keap1 serves as an adapter for cullin 3 (Cul3)/ring-box 1 (Rbx1)-mediated ubiquitination and degradation of Nrf2, which decreases Nrf2 activity [35]. The generation of ROS causes KEAP1 to undergo a conformational change, thus, dissociating from Nrf2, which in turn, translocates to the nucleus. Within the nucleus, it regulates antioxidant response element (ARE)-mediated expression of anti-oxidant enzyme and cytoprotective proteins, including the transcription of downstream target genes, such as $\mathrm{NAD}(\mathrm{P}) \mathrm{H}$ :quinone oxido-reductase 1 (NQO1), heme oxygenase-1 (HO-1), glutathione peroxidase (GPx) and the superoxide dismutase (SOD) family [36, 37] and CAT [31, 38]. 
We therefore tested the hypothesis that Nrf2 activation plays a crucial role in protecting the endothelium from oxidative injury when endothelial cells were treated with palmitate. Our results demonstrate a significant decline in $\mathrm{Nrf} 2$ and its downstream cytoprotective proteins. These findings could be explained in terms of palmitate-induced overproduction of ROS. It has been reported by Mann et al. [39] that the basal activity of NADPH oxidase produces ROS which activate Nrf2/ARE-mediated antioxidant genes in order to maintain redox homeostasis. However, in this acute model, using palmitate, we suggest that excessive ROS production enhances the feedback mechanism and leads to Nrf2 down-regulation. Our hypothesis is based on results of studies demonstrating down-regulation of Nrf2 pathway under oxidative stress conditions. In this context, oscillating high glucose [40] and disturbed shear stress on the vascular wall resulting from oscillatory blood flow [41], diminishes the Nrf2-mediated activation of ARE-linked genes. A high-fat diet also evoked a significant increase in the mRNA abundance of HO-1 in the aortas of Nrf2+/+ mice but not Nrf2-/- mice, suggesting that adaptive activation of the Nrf2/ARE pathway confers endothelial protection [42]. More recently, Fratantonio et al. [43] reported a significantly down-regulated Nrf2, NQO1 and HO-1 expression in HUVECS exposed to palmitic acid for 3 hours, lending support for the findings of this study.

For the first time, we demonstrate that small molecule glycomimetics are able to induce Nrf2 under FFA conditions, thus protecting against oxidative stress. As a consequence of Nrf2 activation, NQO-1 and HO1 gene and protein expression was up-regulated following glycomimetic pretreatment. Multiple studies support the notion that induced expression of Nrf2/ARE-regulated cytoprotective proteins may contribute to the atheroprotective phenotype in endothelial cells $[44,45]$. A negative feedback regulatory loop between NADPH oxidase and Nrf2 has been suggested. Under homeostatic conditions, NOX4 generates superoxide and $\mathrm{H}_{2} \mathrm{O}_{2}$ which activate $\mathrm{Nrf2}$ by inactivating Keap1 [46]. The activated Nrf2 then inhibits the transcription of NOX4 to lower ROS production $[47,48]$. HO-1 reduces oxidative stress-induced cell damage by restoring the balance of antioxidants and pro-oxidants in the vasculature. HO-1 is also known to degrade the prooxidant haem to biliverdin, which is subsequently converted to the radical scavenger bilirubin [49]. By 
interrupting the assembly and activation of the enzyme, bilirubin may directly inhibit NADPH oxidase in endothelial cells, both in vitro and in vivo [50,51]. Hence, Nrf2/HO-1 pathway represents a key adaptive mechanism for moderating the severity of oxidative stress provoked cell damage.

Having demonstrated the protective effects of small molecule glycomimetics on FFA-induced endothelial dysfunction in vitro, we have evaluated their effects on endothelium-dependent vasodilatation and ROS production ex vivo in the presence of palmitate. Mouse aortic rings incubated with palmitate for 24 hours exhibited impaired ACh-induced relaxation. The diminished vasodilatation was associated with increased NADPH oxidase activity and ROS production, confirmed by DHE staining, in palmitate-induced mouse aortic rings, in agreement with Toral et al. [26]. They also reported diminished endothelium-dependent vasodilatation in aortas from high fat diet-fed mice. When the NADPH oxidase inhibitor apocynin and the mitochondrial antioxidant mitoQ were applied, both partially counteracted the palmitate-induced impairment of endothelium-dependent vasodilatation, strengthening the findings of Toral et al. [26], thus confirming activation of oxidative stress pathways. Of note, all tested small molecule glycomimeticis were able to improve endothelium-dependent relaxation, diminish ROS production and decrease activity of NADPH oxidase in mouse aortic rings. Moreover, incubation of the aortic rings with a high concentration of the eNOS inhibitor L-NAME almost abolished the ACh-induced vasodilatation, indicating that it is completely dependent on eNOS-derived NO. These findings suggest that small molecule glycomimetics exert their effects on vascular relaxation, mainly through inhibition of ROS production and an increase in NO bioavailability.

In conclusion, we provide the first evidence that small molecule glycomimetics exert protective effects against FFA-induced endothelial dysfunction. Our mechanistic evidence suggests the involvement of Akt/eNOS and Nrf2/ARE signaling pathways in mediating the effects of glycomimetics, summarized schematically in Figure 9. These chemical probes increased NO bioavailability through their ability to positively regulate Akt and eNOS gene expression as well as protein phosphorylation. In addition, the 
coordinate induction of cytoprotective proteins through activation of the Nrf2/ARE pathway potentially protected the endothelium against FFA-induced oxidative stress. Therefore, small molecule glycomimetics represent a field of great interest for application in the prevention and therapy of FFA-induced endothelial dysfunction as well as for other diseases related to oxidative stress and warrant further investigation.

\section{ACKNOWLEDGMENTS}

The authors wish to thank the Science \& Technology Development Fund (STDF; EGYPT) and the International Atherosclerosis Society (IAS; USA) for supporting the travel of Ayman Mahmoud. The authors thank Faye Owen and Nicola Neale for technical assistance.

\section{DISCLOSURES}

The authors and Manchester Metropolitan University have a commercial interest in glycomimetics C1-C4 and their method of use (UK Patent Application No. 1515850.4 in the name of Manchester Metropolitan University).

\section{REFERENCES}

[1] Moncada S, Higgs EA. Nitric oxide and the vascular endothelium. Handb Exp Pharmacol. 2006; (176 Pt 1): 213-54.

[2] Vanhoutte PM, Shimokawa H, Feletou M, Tang EH. Endothelial dysfunction and vascular disease - a thirthieth anniversary update. Acta Physiol (Oxf). 2015. doi: 10.1111/apha.12646.

[3] Rodriguez-Manas L, Angulo J, Vallejo S, Peiro C, Sanchez-Ferrer A, Cercas E, Lopez-Doriga P, et al. Early and intermediate amadori glycosylation adducts, oxidative stress, and endothelial dysfunction in the streptozotocin-induced diabetic rats vasculature. Diabetologia. 2003 ; 46: 55666. 
[4] Feletou M, Vanhoutte PM. Endothelial dysfunction: A multifaceted disorder (the wiggers award lecture). Am J Physiol Heart Circ Physiol. 2006; 291: H985-1002.

[5] Tousoulis D, Papageorgiou N, Androulakis E, Siasos G, Latsios G, Tentolouris K, et al. Diabetes mellitus-associated vascular impairment: Novel circulating biomarkers and therapeutic approaches. J Am Coll Cardiol. 2013; 62: 667-76.

[6] Forst T, Weber MM, Pfutzner A. Cardiovascular benefits of GLP-1-based herapies in patients with diabetes mellitus type 2: Effects on endothelial and vascular dysfunction beyond glycemic control. Exp Diabetes Res. 2012; 2012: 635472.

[7] Rask-Madsen C, King GL. Mechanisms of disease: Endothelial dysfunction in insulin resistance and diabetes. Nat Clin Pract Endocrinol Metab. 2007; 3: 46-56.

[8] Poynten AM, Gan SK, Kriketos AD, Campbell LV, Chisholm DJ. Circulating fatty acids, non-high density lipoprotein cholesterol, and insulin-infused fat oxidation acutely influence whole body insulin sensitivity in nondiabetic men. J Clin Endocrinol Metab. 2005; 90: 1035-40.

[9] Rask-Madsen C, Kahn CR. Tissue-specific insulin signaling, metabolic syndrome, and cardiovascular disease. Arterioscler Thromb Vasc Biol. 2012; 32: 2052-9.

[10] Wu Y, Zhou H, Wu K, Lee S, Li R, Liu X. PTEN phosphorylation and nuclear export mediate free fatty acid-induced oxidative stress. Antioxid Redox Signal. 2014; 20: 1382-95.

[11] Zhang D, Gao X, Wang Q, Qin M, Liu K, Huang F, et al. Kakkalide ameliorates endothelial insulin resistance by suppressing reactive oxygen species-associated inflammation. J Diabetes. 2013; 5: 13-24.

[12] Zhang H, Dellsperger KC, Zhang C. The link between metabolic abnormalities and endothelial dysfunction in type 2 diabetes: An update. Basic Res Cardiol. 2012; 107: 237.

[13] Lee CH, Lee SD, Ou HC, Lai SC, Cheng YJ. Eicosapentaenoic acid protects against palmitic acid-induced endothelial dysfunction via activation of the AMPK/eNOS pathway. Int J Mol Sci. 2014; 15: 10334-49. 
[14] Bishop JR, Schuksz M, Esko JD. Heparan sulphate proteoglycans fine-tune mammalian physiology. Nature. 2007; 446: 1030-7.

[15] Turnbull J, Powell A, Guimond S. Heparan sulfate: Decoding a dynamic multifunctional cell regulator. Trends Cell Biol. 2001; 11: 75-82.

[16] Lortat-Jacob H. The molecular basis and functional implications of chemokine interactions with heparan sulphate. Curr Opin Struct Biol. 2009; 19: 543-8.

[17] Tyler PC, Guimond SE, Turnbull JE, Zubkova OV. Single-entity heparan sulfate glycomimetic clusters for therapeutic applications. Angew Chem Int Ed Engl. 2015; 54: 2718-23.

[18] Magnani JL, Ernst B. Glycomimetic drugs--a new source of therapeutic opportunities. Discov Med. 2009; 8: 247-52.

[19] Jones AM, Lebl T, Patterson S, van Mourik T, Früchtl HA, Philp D, et al. Parallel synthesis and spectroscopic analysis of a collection of heterocycles based on the diazabenz[e]aceanthrylene core structure. Tetrahedron. 2009; 65: 563-78.

[20] Raiber EA, Wilkinson JA, Manetti F, Botta M, Deakin J, Gallagher J, et al. Novel heparin/heparan sulfate mimics as inhibitors of HGF/SF-induced MET activation. Bioorg Med Chem Lett. 2007; 17: 6321-5.

[21] Jacobsen EN, Marko I, Mungall WS, Schroeder G, Sharpless KB. Asymmetric dihydroxylation via ligand-accelerated catalysis. J Am Chem Soc. 1988; 110: 1968-70.

[22] Lipinski CA, Lombardo F, Dominy BW, Feeney PJ. Experimental and computational approaches to estimate solubility and permeability in drug discovery and development settings. Adv Drug Deliv Rev. 1997; 23: 3-25.

[23] Chavez JA, Summers SA. Characterizing the effects of saturated fatty acids on insulin signaling and ceramide and diacylglycerol accumulation in 3T3-L1 adipocytes and $\mathrm{C} 2 \mathrm{C} 12$ myotubes. Arch Biochem Biophys. 2003; 419: 101-9. 

PPAR $\beta$ activation restores the high glucose-induced impairment of insulin signalling in endothelial cells. Br J Pharmacol. 2014; 171: 3089-102.

[25] Romero M, Jimenez R, Sanchez M, Lopez-Sepulveda R, Zarzuelo MJ, O'Valle F, et al. Quercetin inhibits vascular superoxide production induced by endothelin-1: Role of NADPH oxidase, uncoupled eNOS and PKC. Atherosclerosis. 2009; 202: 58-67.

[26] Toral M, Romero M, Jimenez R, Mahmoud AM, Barroso E, Gomez-Guzman M, et al. Carnitine palmitoyltransferase-1 up-regulation by PPAR- $\beta / \delta$ prevents lipid-induced endothelial dysfunction. Clin Sci (Lond). 2015; 129: 823-37.

[27] Romero M, Jimenez R, Sanchez M, Lopez-Sepulveda R, Zarzuelo A, Tamargo J, et al. Vascular superoxide production by endothelin-1 requires Src non-receptor protein tyrosine kinase and MAPK activation. Atherosclerosis. 2010; 212: 78-85.

[28] Magnani JL. Glycomimetic drugs: A source of novel therapeutics. In: Endo T, Seeberger HP, Hart WG, Wong C-H, Taniguchi N, eds. Glycoscience: Biology and medicine. Tokyo: Springer Japan:1-6.

[29] Kuzkaya N, Weissmann N, Harrison DG, Dikalov S. Interactions of peroxynitrite, tetrahydrobiopterin, ascorbic acid, and thiols: Implications for uncoupling endothelial nitric-oxide synthase. J Biol Chem. 2003; 278: 22546-54. to restore renal nitric oxide and prevent hypertension in young spontaneously hypertensive rats: Lcitrulline and nitrate. Transl Res. 2014; 163: 43-52.

[31] Inoguchi T, Li P, Umeda F, Yu HY, Kakimoto M, Imamura M, et al. High glucose level and free fatty acid stimulate reactive oxygen species production through protein kinase C-dependent activation of NAD(P)H oxidase in cultured vascular cells. Diabetes. 2000; 49: 1939-45.

[32] Brownlee M. The pathobiology of diabetic complications: A unifying mechanism. Diabetes. 2005; 54: 1615-25. 

of oxidative stress, endoplasmic reticulum stress, and c-Jun n-terminal kinase in pancreatic betacell dysfunction and insulin resistance. Int J Biochem Cell Biol. 2006; 38: 782-93.

[34] Leopold JA, Loscalzo J. Oxidative enzymopathies and vascular disease. Arterioscler Thromb Vasc Biol. 2005; 25: 1332-40.

[35] Niture SK, Khatri R, Jaiswal AK. Regulation of Nrf2-an update. Free Radic Biol Med. $2014 ; 66: 36-44$.

[36] Cho HY, Kleeberger SR. Nrf2 protects against airway disorders. Toxicol Appl Pharmacol. 2010; 244: 43-56.

[37] Hayes JD, Dinkova-Kostova AT. The Nrf2 regulatory network provides an interface between redox and intermediary metabolism. Trends Biochem Sci. 2014; 39: 199-218.

[38] Jaiswal AK. Nrf2 signaling in coordinated activation of antioxidant gene expression. Free Radic Biol Med. 2004; 36: 1199-207.

[39] Mann GE, Rowlands DJ, Li FY, de Winter P, Siow RC. Activation of endothelial nitric oxide synthase by dietary isoflavones: Role of NO in Nrf2-mediated antioxidant gene expression. Cardiovasc Res. 2007; 75: 261-74.

[40] Liu TS, Pei YH, Peng YP, Chen J, Jiang SS, Gong JB. Oscillating high glucose enhances oxidative stress and apoptosis in human coronary artery endothelial cells. J Endocrinol Invest. 2014; $37: 645-51$.

[41] Cheng X, Siow RC, Mann GE. Impaired redox signaling and antioxidant gene expression in endothelial cells in diabetes: A role for mitochondria and the nuclear factor-E2-related factor 2kelch-like ECH-associated protein 1 defense pathway. Antioxid Redox Signal. 2011; 14: 469-87. Ungvari Z, Bailey-Downs L, Gautam T, Jimenez R, Losonczy G, Zhang C, et al. Adaptive induction of NF-E2-related factor-2-driven antioxidant genes in endothelial cells in response to hyperglycemia. Am J Physiol Heart Circ Physiol. 2011; 300: H1133-40. 

endothelial dysfunction is attenuated by cyanidin-3-O-glucoside through modulation of Nrf2/Bach1 and NF-kB pathways. Toxicol Lett. 2015; 239: 152-60.

[44] Chen XL, Dodd G, Thomas S, Zhang X, Wasserman MA, Rovin BH, Kunsch C. Activation of nrf2/are pathway protects endothelial cells from oxidant injury and inhibits inflammatory gene expression. Am J Physiol Heart Circ Physiol. 2006; 290: H1862-70.

[45] Speciale A, Anwar S, Ricciardi E, Chirafisi J, Saija A, Cimino F. Cellular adaptive response to glutathione depletion modulates endothelial dysfunction triggered by TNF- $\alpha$. Toxicol Lett. 2011; 207: 291-7.

[46] Brewer AC, Murray TV, Arno M, Zhang M, Anilkumar NP, Mann GE, et al. Nox4 regulates Nrf2 and glutathione redox in cardiomyocytes in vivo. Free Radic Biol Med. 2011; 51: $205-15$.

[47] Goettsch C, Goettsch W, Brux M, Haschke C, Brunssen C, Muller G, et al. Arterial flow reduces oxidative stress via an antioxidant response element and Oct-1 binding site within the NADPH oxidase 4 promoter in endothelial cells. Basic Res Cardiol. 2011; 106: 551-61. Nrf2 regulates ROS production by mitochondria and NADPH oxidase. Biochim Biophys Acta. 2015; 1850: 794-801.

[49] Siow RC, Sato H, Mann GE. Heme oxygenase-carbon monoxide signalling pathway in atherosclerosis: Anti-atherogenic actions of bilirubin and carbon monoxide? Cardiovasc Res. 1999; 41: 385-94.

[50] Jiang F, Roberts SJ, Datla, Sr., Dusting GJ. NO modulates NADPH oxidase function via heme oxygenase-1 in human endothelial cells. Hypertension. 2006; 48: 950-7.

[51] Datla SR, Dusting GJ, Mori TA, Taylor CJ, Croft KD, Jiang F. Induction of heme oxygenase-1 in vivo suppresses NADPH oxidase derived oxidative stress. Hypertension. 2007; 50: 636-42. 
Figure legends:

Figure 1. The four glycomimetics C1-C4 synthesized and used in this study.

Figure 2. Glycomimetics rescue palmitate-induced impairment of NO production. HUVECS incubated with $1 \mu \mathrm{mol} / \mathrm{L}$ of the glycomimetics C1-C4 in the presence of palmitate for 3 hours (A) or pretreated with C1-C4 for 12 hours followed by 3 hours palmitate (B). Treatment with either $1 \mu \mathrm{mol} / \mathrm{L}$ C1-C4 for 3 hours along with palmitate or for 12 hours followed by the addition of palmitate for 3 hours protects against palmitate-induced reduction in NO production. Results are mean $\pm \mathrm{SEM} ; \mathrm{N}=8-12$. ${ }^{*} * * \mathrm{P}<0.01$ versus control, and $\# \mathrm{P}<0.05, \# \# \mathrm{P}<0.01$ and $\# \# \# \mathrm{P}<0.001$ versus $\mathrm{PAL}$. PAL, palmitate; AUC, area under curve; DAF-2, diaminofluorescein-2.

Figure 3. Glycomimetics modulate mRNA abundance and protein phosphorylation of eNOS and Akt under palmitate-induced oxidative stress. HUVECs were pretreated with C1-C4 for 12 hours followed by 3 hours of palmitate. Treatment with palmitate decreases both Akt mRNA (A) and protein phosphorylation (B), and reduces mRNA (C) and phosphorylation of eNOS (D). All tested glycomimetics at $1 \mu \mathrm{mol} / \mathrm{L}$ concentration up-regulate Akt and eNOS mRNA abundance and phosphorylation in HUVECs treated with palmitate. Results are mean $\pm \mathrm{SEM} ; \mathrm{N}=6$. ${ }^{*} \mathrm{P}<0.05$ and ${ }^{* *} \mathrm{P}<0.01$ versus control, and $\# \mathrm{P}<0.05$, $\# \# \mathrm{P}<0.01$ and \#\#\#P<0.001 versus $\mathrm{PAL}$. PAL, palmitate; Akt, protein kinase $\mathrm{B}$; eNOS, endothelial nitric oxide synthase.

Figure 4. Glycomimetics reduce palmitate-induced ROS production and oxidative stress. Glycomimetics exhibit protective effect against palmitate-induced ROS production in HUVECS either incubated with $1 \mu \mathrm{mol} / \mathrm{L}$ C1-C4 in the presence of palmitate for 3 hours $(\mathbf{A})$ or pretreated with C1-C4 for 12 hours followed by 3 hours palmitate (B). HUVECs treated with palmitate for 3 hours show significant increase in MDA, a marker of lipid peroxidation $(\mathbf{C})$, and concomitant decrease in the activity of SOD (D) and CAT (E). Pretreatment with $1 \mu \mathrm{mol} / \mathrm{L}$ C1-C4 prevent palmitate-induced lipid peroxidation and diminished activity of antioxidant enzymes in HUVECs. Results are mean $\pm \mathrm{SEM} ; \mathrm{N}=6-10 .{ }^{* *} \mathrm{P}<0.01$ and $* * * \mathrm{P}<0.001$ versus control, and $\# \mathrm{P}<0.05, \# \# \mathrm{P}<0.01$ and \#\#\#P<0.001 versus $\mathrm{PAL}$. PAL, palmitate; ROS, reactive oxygen species; MDA, malondialdehyde, $\mathrm{SOD}$, superoxide dismutase; $\mathrm{CAT}$, catalase.

Figure 5. Glycomimetics modulate NADPH oxidase activity in HUVECs in response to palmitate. HUVECS pretreated with C1-C4 for 12 hours followed by 3 hours palmitate. (A) NADPH oxidase activity measured by lucigenin-enhanced chemiluminescence. (B-E) Expression of NADPH oxidase subunits NOX1, NOX4, p2 $2^{\text {phox }}$ and $\mathrm{p} 47^{\text {phox }}$ at the level of mRNA by RT-PCR. Palmitate increases both NADPH oxidase activity and expression of the enzyme subunits. C1-C4 reduce palmitate-induced NADPH oxidase activation and expression in HUVECs treated 12 hours with the addition of palmitate for 3 hours. Results are mean $\pm \mathrm{SEM} ; \mathrm{N}=6-8 .{ }^{*} * \mathrm{P}<0.01$ and $* * * \mathrm{P}<0.001$ versus control, and $\# \mathrm{P}<0.05, \# \# \mathrm{P}<0.01$ and $\# \# \# \mathrm{P}<0.001$ versus PAL. PAL, palmitate; NADPH oxidase, nicotinamide adenine dinucleotide phosphate oxidase.

Figure 6. Glycomimetics protect against palmitate-induced oxidative stress via the Nrf2/ARE pathway. The effect of C1-C4 and palmitate treatment on HUVECs with regard to Nrf2 (A \& B), NQO-1 $(\mathbf{C} \& \mathbf{D})$ and HO-1 (E \& F) levels was determined using qRT-PCR and western blotting. Treatment with palmitate produces a significant decrease in both mRNA and protein levels of Nrf2, NQO-1 and HO-1. Pretreatment with $1 \mu \mathrm{mol} / \mathrm{L}$ C1-C4 increases levels of Nrf2, NQO-1 and HO-1 in HUVECs treated with palmitate. Results are mean $\pm \mathrm{SEM} ; \mathrm{N}=6 .{ }^{*} \mathrm{P}<0.05, * * \mathrm{P}<0.01$ and $* * * \mathrm{P}<0.001$ versus control, and $\# \mathrm{P}<0.05$, \#\#P<0.01 and \#\#\#P<0.001 versus $\mathrm{PAL}$. PAL, palmitate; Nrf2, nuclear factor-erythroid 2-related factor 2; NQO-1, NAD(P)H:quinone oxido-reductase 1; HO-1, heme oxygenase-1. 
Figure 7. Glycomimetics restore endothelium-dependent vasodilatation ex vivo. Mice aortic rings were incubated with $100 \mu \mathrm{mol} / \mathrm{L}$ palmitate and 1 or $10 \mu \mathrm{mol} / \mathrm{L}$ of $(\mathbf{A}) \mathbf{C 1},(\mathbf{B}) \mathbf{C 2},(\mathbf{C}) \mathbf{C 3}$ or (D) $\mathbf{C 4}$ for 24 hours. Concentration-relaxation response curves to ACh were performed in intact rings pre-contracted by U46619. Treatment of aortic rings with palmitate for 24 hours diminished endothelium-dependent vasodilator responses to ACh, an effect that was significantly reversed by either dose of the glycomimetics C1-C4. (E $\&$ F) ROS are involved in endothelial dysfunction induced by palmitate. MitoQ and apocynin improve the impaired aortic relaxation in response to $\mathrm{ACh}$, induced by palmitate. Results are mean $\pm \mathrm{SEM} ; \mathrm{N}=8-12$.

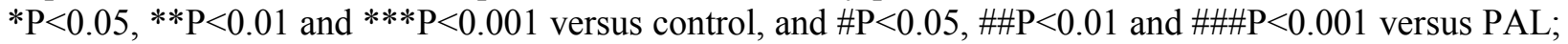
$\mathrm{CT}$, control; PAL, palmitate; ACh, acetylcholine.

Figure 8. Glycomimetics reduce vascular ROS levels by reducing NADPH oxidase activity ex vivo. (A) aortic rings were incubated with $100 \mu \mathrm{mol} / \mathrm{L}$ palmitate and 1 or $10 \mu \mathrm{mol} / \mathrm{L}$ of $\mathbf{C 1}-\mathbf{C} 4$ for 24 hours and the level of red fluorescence was measured in sections of aorta incubated with DHE. Treatment of aortic rings with palmitate for 24 hours increased staining in adventitial, medial and endothelial cells. Coincubation of rings with either 1 or $10 \mu \mathrm{mol} / \mathrm{L} \mathbf{C 1}-\mathbf{C} 4$ produced a significant $(\mathrm{P}<0.001)$ reduction in the red fluorescence. (B) NADPH oxidase activity increased in aortic rings treated palmitate for 24 hours. Both doses of C1-C4 reduce palmitate-induced NADPH oxidase activity. Results are mean \pm SEM; $\mathrm{N}=6$. $* * * \mathrm{P}<0.001$ versus control, and $\# \# \mathrm{P}<0.01$ and \#\#\#P<0.001 versus $\mathrm{PAL}$. PAL, palmitate; NADPH oxidase, nicotinamide adenine dinucleotide phosphate oxidase; DHE, dihydroethidium. Scale bar $=50 \mu \mathrm{m} . \mathrm{X} 400$.

Figure 9. Schematic diagram demonstrating the protective effects of small molecule glycomimetics against FFA-induced endothelial dysfunction. FFA, free fatty acid; Akt, protein kinase B, eNOS, endothelial nitric oxide synthase; NO, nitric oxide; Nrf2, nuclear factor-erythroid 2-related factor 2; NQO1, $\mathrm{NAD}(\mathrm{P}) \mathrm{H}$ :quinone oxido-reductase 1 ; $\mathrm{HO}-1$, heme oxygenase-1; $\mathrm{O}_{2}{ }^{--}$, superoxide radical; ONOO', peroxynitrite; GPx; glutathione peroxidase; GST, glutathione-S-transferase; SOD, superoxide dismutase; CAT, catalase; ARE, antioxidant response element. 